\title{
Look before you LEAPPTM: An interprofessional approach to bedside pediatric inpatient procedures
}

\author{
Ann S Botash, Michelle Jeski, Colleen Baish Cameron, Elizabeth K. Nelsen, Pamela Haines, Nicholas Bennett
}

\begin{abstract}
The Golisano Children's Hospital at Upstate Medical University is a 71 bed children's hospital within a hospital, serving nearly two million people in Central New York. Minor procedures occur daily in all children's hospitals, yet team coordination when planning for these procedures is often overlooked. LEAPPTM is a mnemonic for: Listen, Evaluate, Anticipate, Plan, and Proceed. The "Look before You LEAPPTM" program was developed by a group of nurses, child life specialists, faculty, a chief resident and a fellow. LEAPPTM is a team-based program providing consistent care to all children undergoing inpatient procedures. It improves patient satisfaction and reduces procedural distress. Through LEAPPTM steps, teams are created at point of care - at the bedside or treatment room of inpatient units. Educational goals are linked to the practical goal of cooperation for good health care.
\end{abstract}

The approach uses an online educational module for residents, students and nurses to introduce an innovative protocol and a planning tool.1 Pocket cards, promotional pens, and logo door-clings, purchased through grant funds (The Foundation for Upstate Medical University) were initially used to encourage participation. Pre/post observations of procedural planning and performance of the nurse, caregiver, physician, childlife specialist and independent observer included patient and family preparation, pain and anxiety, staffing and supplies, and satisfaction. Fifty procedures were assessed pre-implementation and 28 post implementation. Although satisfaction with procedures improved between pre and post LEAPPTM implementation, there were overall differences in satisfaction with procedural management and pain/anxiety control by physicians, caregivers, and staff that remained statistically significant. Interdisciplinary bedside teamwork can be used to support interprofessional education and this education can similarly be used to support improved patient outcomes.

\section{Problem}

Each day, hospitalized children undergo a range of minor procedures, such as peripheral lines, blood draws, dressing changes, wound and burn care, incision and drainage, packing removals, lumbar punctures, "bedside" scoping or other procedures. Each of these procedures has the potential to cause emotional trauma to the child and family. Although caregivers, physicians and staff are empathetic and compassionate, provider attitudes and behaviours are not enough.

Child life specialists and nurses anecdotally identified the problem of lack of coordination of inpatient bedside procedures. Situations were observed where resident and attending physicians would perform procedures at the bedside without notifying the child's nurse of the plan. In some cases, the parents were also not present or notified. There were other situations where patients were awakened from sleep so that a procedure could be performed.

In some cases, procedures were performed with partially implemented best practices for utilizing child life services or nursing services to assist with procedures and in others there was a disorganized approach to procedures with no clear plan for what to do when things go wrong. Misuse of the "bedside" for invasive procedures, rather than the treatment room was also an issue. Upstate's pre-existing policy for invasive procedures was not written for bedside procedures and found to be vague and insufficient. There were no clear written guidelines for procedural pain. Assessment and planning stages for procedures were often not present and the practices did not meet institutional standards or values for patient care. There was a sense that it was common for these procedures to be poorly tolerated by patients. They often had to be rescheduled in order to ask for non-pharmacologic pain and anxiety management assistance (as from child life specialists) or sedation.

Collaboration between nurses and other health care professionals for paediatric procedural management at point of care was anticipated to improve patient satisfaction and reduce procedural distress. This concept involves reduction of procedural distress through assessment of the family's concerns, prior patient experiences with procedures, provider preparation, and adequate control of pain and anxiety during the procedure. Standards within one protocol for this type of management have not been developed nor reported in the literature.

\section{Background}

Evidence has shown bedside procedures can be traumatic and use of the treatment room, when available, is a better option for more invasive procedures.2 Family or parental presence during procedures seem to benefit both family and patient. Staff can have an opportunity to listen to parent concerns and evaluate and anticipate patient needs. 3

Using a preparation model, a task force at the Golisano Children's Hospital reviewed the literature and combined best practices to create a single model to address inpatient paediatric procedure 
management. LEAPPTM is an acronym for Listen, Evaluate, Anticipate, Plan and Proceed. The mnemonic is based on procedural phases (anticipation, preparation, procedural, recovery)2 and mapped to integral interprofessional educational competencies (communication and teamwork)3. This report will describe the development, implementation and effect of LEAPPTM on paediatric inpatient procedure management.

\section{Baseline Measurement}

To ensure a team would be readily available, staff and child life specialists focused efforts on early identification of procedures and communication with physicians prior to procedure. All participants in a particular procedure (nurse, caregiver, physician, child life specialist and independent observer) were surveyed after the procedure with regard to preparation, pain and anxiety, staffing and supply adequacy, and overall satisfaction with the procedure. Fifty medical procedures were assessed using a 4-point Likert scale (strongly disagree, disagree, agree, strongly agree) asking questions on the preparation, medical and non-medical interventions, pain control and level of support available for the procedure (see details below in Design). The feedback from these baseline surveys demonstrated most residents, faculty and staff did not communicate well prior to performing a bedside procedure. All physicians surveyed agreed $(24.4 \%)$ or strongly agreed $(75.6 \%)$ pain and anxiety were well controlled during the procedure, compared to $55 \%$ of caregivers and $60 \%$ of staff $(p<0.01$ ). Physicians rated their overall procedural management higher when compared to staff and caregivers. Close to $72 \%$ of physicians strongly agreed the procedure was successful as compared to $60 \%$ of caregivers and $52 \%$ of staff $(p<0.01)$. This discrepancy between staff and caregivers was felt to be due to physicians being unaware of the value or need for coordination of efforts. Aside from clear differences in perception between providers and others, the results also highlighted the fact that caregivers and staff were often less satisfied with how the procedure was performed.

\section{Design}

A group of interested staff, faculty, chief residents, and a fellow met to brainstorm ways to address problems identified on a regular basis through direct observation of poorly orchestrated procedures on the paediatric units. This group designed, implemented and studied the project.

Phases of anticipation, preparation, procedural, and recovery were identified and used as a basis for the development of a method to teach interprofessional approaches to procedure management. Best practices guidelines were studied. 4, 5

The mnemonic, LEAPPTM, was used to assist with the steps of a newly designed process. See Table 1. Specifics were defined in a PowerPoint presentation and a 10 minute video (http://www.youtube.com/watch?v=T2f7G6zMdXA ) created to teach residents and students. Actors and narrators in the video are respected faculty of various paediatric subspecialties, including the surgical subspecialties. Support from departmental leaders was felt to be an important aspect in enabling project success. The LEAPPTM mnemonic was described in a card format, given to residents who completed the video followed by a 5 question postvideo quiz. A frog logo was designed and used to represent LEAPPTM. The logo was utilized in a plastic format("cling") to be placed on patient room doors reminding staff of a potential procedural need. The logo remained in place for patients with frequent procedures and/or known distressful responses to previous procedures. LEAPPTM pens were distributed at the initial introduction of the program to create an incentive for completion of the online video and quiz.

The educational materials became part of mandatory orientation of all residents (over 500 residents and fellows each year) at Upstate Medical University in 2010. It also became a component of the paediatric clerkship curriculum. Education later included multiple educational sessions with various subspecialties, grand rounds in nursing and paediatric and presentations at new resident orientations in surgery and paediatric residencies. Nurses and child life specialists received similar required education online and in print form. Hospital policies and planning tools were developed and accepted by hospital administration. A pre-procedural assessment tool was created by nursing staff, and once complete, it was placed in the chart as a means to provide a central location for those involved in the anticipated procedure. Nurses were encouraged to use the tool, even without a formal order. Nurses and child life specialists took the lead in the inpatient setting to take steps to promote the process. Faculty and residents implemented the process with guidance from staff.

Real-time observation tools were created to survey the nurse, caregiver, physician, child life specialist and independent observer regarding procedural planning and performance. All participants in a procedure were surveyed after the procedure with regard to preparation of patient and family if pain and anxiety were assessed and addressed, if adequate staffing and supplies were in the treatment room, and overall satisfaction with the procedure. Students who were not part of the clinical team performed direct observation as part of an elective for their summer research experience (see acknowledgments below). Pre and post observations of procedures were compared over an 18-month period using custom software (Samurai Scientific, Syracuse, NY) which organized and collated the survey responses by respondent and question. The frequency of responses in each category were compared between groups and the statistical significance of any differences assessed using Chi Squared test, with $p<0.01$.

Categories containing fewer than five responses were merged with the adjacent category to ensure validity. No responses in the "Disagree" category were merged into the "Agree" category. Success was measured throughout pre and post analysis. See Table 2 listing of survey questions.

\section{Strategy}

\section{PDSA Cycle 1}

The initial test run of our intervention demonstrated several issues that needed to be addressed. We noted immediately some 
residents and fellows of certain sub specialties did not follow the new protocol and had to be reminded. The LEAPPTM designers took steps to address this through notification of the residency program directors and chairs of these departments. In order to adequately track the procedures, LEAPPTM nursing champions were identified on each unit. These nurses were charged with providing survey forms for completion. For the second cycle (follow-up surveys after implementation) nurses were charged with being aware of upcoming procedures, filling out planning tools, and anticipating and facilitating teamwork for the procedures.

Early in the implementation of the program, it became apparent that we needed to have resources available in each of the treatment rooms. A resource manual was developed and placed in each inpatient treatment room. The manual contained a copy of the Power Point presentation, invasive guidelines policy, interventions for pain and anxiety management, pictorials for the use of therapeutic holds (restraining methods), resources on developmental aspects of procedural distress and information for caregivers on child coping strategies.

PDSA 2

Although we did not have data regarding numbers of procedures performed on the inpatient units, there was a general consensus we were now forming point-of-care teams for almost every procedure. Procedures not performed according to the LEAPPTM steps were now outliers. Resident and nurse verbal informal feedback indicated that in most cases, the team had accepted the new protocol and followed the steps.

\section{Post-Measurement}

Follow-up data showed most physicians agreed or strongly agreed (96\%) pain and anxiety were well controlled, $84.2 \%$ of caregivers and $92.6 \%$ of staff agreed or strongly agreed $(p=0.4549)$. However, overall differences in satisfaction with procedural management by physicians, caregivers, and staff remained statistically significant. Physicians still rated overall satisfaction with procedural management higher after implementation (99.2\%) than caregivers $(93.8 \%)$ and staff $(96 \%, p=0.0045)$. LEAPPTM implementation led to improved correlation among staff, caregivers, and physicians with regard to observations on pain and anxiety control and overall procedure satisfaction.

The overall impressions from caregivers remained similar postintervention but those from staff increased. This may reflect a lack of awareness of "behind the scenes" work in gathering support, or perhaps not being aware of how an ideal procedure should proceed. In addition, although the overall positive responses from providers remained high post-intervention, there was a trend towards more "agree" responses rather than "strongly agree", perhaps due to awareness through education and training of expectations. For example, pre-implementation $73.3 \%$ of providers strongly agreed there was sufficient evaluation of children prior to the procedure, compared to $42.9 \%$ post-LEAPPTM. Similarly, perception of needs for pre-medication $(70.5 \%$ vs. $50 \%)$, whether all needs were met $(73.3 \%$ vs. $50 \%)$ and whether there was sufficient preparation $(71.1 \%$ vs. $48 \%)$ were lower in the "strongly agree" category, even though overall positive responses remained between $95 \%-100 \%$.

In the three years since implementation of this process there continue to be occasional incidents where patient bedside procedures are not well coordinated, however these are the exception, rather than the rule. There were weekly LEAPPTM meetings and twice per month LEAPPTM rounds for one year post implementation. This was followed by monthly safety rounds where LEAPPTM and procedure issues are discussed. The use of these policies, planning tools and door clings has functioned as a guide and reminder to staff and physicians so that we now have a culture of a team approach to inpatient procedures.

See supplementary file: ds2222.docx - "Table 1 (LEAPPTM steps) and Table 2 (Survey questions)"

\section{Lessons and Limitations}

One limitation of the study was the reduced numbers of completed post-implementation surveys. This was likely due to a decrease in enthusiasm for data collection over time. The process had continued beyond the time frame during which students were available to assist with data collection and independent observation.

Implementation was challenged by having to teach and re-teach various clinical services. Residents would rotate frequently and, although they had completed the online module, it may have been months prior to their rotation on the paediatric service. In addition the variety of procedures also required adaptation of procedural management, for example, orthopaedics and ear-nose-throat surgeons perform very different procedures and therefore standardization of the process was difficult. If we were to repeat this effort, we would utilize more departmental leaders to be core members of the initial developmental process to assist with development of more specific processes depending on procedures. This would also have improved earlier buy-in. The program identified other gaps in the system, such as the need for a standard pain treatment protocol and procedure focused patient education handouts. We recommend developing this prior to project implementation.

\section{Conclusion}

Interdisciplinary approaches to patient care can be implemented through a multifaceted approach, including education of residents, students, nurses and faculty, development of policies and tools, use of point of care reminders and ongoing surveillance of employed method. Although there are still occasional situations where physicians have been observed to perform a procedure without the team approach, it is now the exception. It was sometimes challenging to get individual physicians to understand the value of the team approach and the data was helpful in convincing them. The promotion of the process by respected faculty colleagues was critical to acceptance of the process. Promoting interdisciplinary bedside teamwork can similarly be used to support improved 


\section{BMJ Quality Improvement Reports}

patient outcomes as well as interprofessional education competencies.6, $7 \mathrm{~A}$ three-pronged quality improvement approach, including development of guidelines, implementation of educational interventions, and strong leadership support, was successful and resulted in development of a best practice and stronger team-based procedural management. The data suggest that we were able to not only improve the care of children undergoing invasive procedures, but that we raised the expectations and understanding of the process across the institution.

\section{References}

1. Botash AS, Nelsen EK, Haines P, Jeski M, Baish C. Look before you LEAPPTM: An interprofessional team approach to inpatient "bedside" pediatric procedures. MedEd Portal 2012;

https://www.mededportal.org/icollaborative/resource/546

2. Fanurik D, Schmitz M, Reach K, Haynes K, Leatherman I. Hospital room or treatment room for pediatric inpatient procedures: which location do parents and children prefer? Pain Res Man 2000; 5(2): 148- 156

3. Boudreaux ED, Francis JL, Loyacano T. Family presence during invasive procedures and resuscitations in the emergency department: A critical review and suggestions for future research. Ann Emerg Med 2002; 40(2): 193-205

4. Cohen L. Behavioral approaches to anxiety and pain management for pediatric venous access. Pediatrics 2008; 122: S134

5. American Academy of Pediatrics. Committee on Psychological Aspects of Child and Family Health; Task Force on Pain in Infants, Children and Adolescents. The assessments and management of acute pain in infants, children and adolescents.Pediatrics 2001; 108(3): 793-797

6. Report of an Expert Panel: Interprofessional Education Collaborative. Core Competencies for Interprofessional Collaborative Practice. May 2011; http://www.aacn.nche.edu/educationresources/ipecreport.pdf

7. Englander R, Cameron T, Ballard AJ, Dodge J, Bull J, Aschenbrener CA. Toward a common taxonomy of competency domains for health professions and competencies for physicians. Acad Med.

2013;88(8):1088-1094

Dr. Nicholas Bennett is part owner of Samurai Scientific, Syracuse, NY, the company utilized to develop our survey database. There are no other conflicts of interest.

\section{Acknowledgements}

Regina Lozito, CCLS is acknowledged for her assistance with coordination, helpful advice and care of patients. Allison Martin, MD and Christine Crana were medical students who provided assistance and served as independent observers for the project during implementation of the LEAPPTM program.
The Foundation for Upstate Medical University provided partial funding support. 